\title{
Ontology based Semantic Web Technologies in E-learning Environment using Protégé
}

\author{
N. Manickasankari*, D. Arivazhagan and G. Vennila \\ Department of Information Technology, AMET University, Chennai, India; \\ manickasankari@gmail.com, arivazhagand@hotmail.com,g.vennila265@gmail.com
}

\begin{abstract}
The rapid advanced development of mobile phone usage has been increased very sharply. A semantic web is major concept about creation of Meta data. Most of the web page oriented application like HTML and XML uses the concept of Meta data. This paper proposes class concept hierarchy method for implementing and retrieving information about e-learning domain through PROTÉGÉ tools and also shows RDFS and OWL based samples method implementation for e-learning domains in these tools. It produced the result like number of course material prepared by tutor, total number of student registration and number of exam, quiz, assignment conducted by particular tutor using SPARQL query language.
\end{abstract}

\section{Introduction}

E learning is a way of learning the information through electronic medium. Web based learning is much prevalent because of the learner learn essential content at anytime, anywhere. Semantic web is a central research area of artificial intelligence to generate data about data (Meta Data). The data should be denoted by RDF, RDFS, OWL based Techniques. These types of tools are support any type of web oriented application. The main challenge of the semantic web is to present well defined meaning, understandable for different end user. Semantic web is also containing the jargon details of all the resources. Semantic Web is built for human and is based on machine readable not by human ${ }^{1}$.

The main usage of semantic web is reusability. This feature allows the user can apply any procedure on particular data. Ontology was familiarized in our model to support the conceptualization of certain domain. Ontology language is based on the web technology standards ${ }^{2}$.

\section{Semantic Web Architecture}

The Figure 1.1 describes the architecture of Semantic web $^{3}$.

The component of the Semantic web technology is based on XML(Extensible Markup Language), XML Schema, RDF(Resource Description Frame Work), RDFS, Web ontology Language (OWL). All these components are based on the XML syntax. The architecture of semantic web includes the details of URI, XML, RDF, and ONTOLOGY. URI is the main component of semantic web layer, and is used to identify the resource like webpage, country code etc. XML and JAVA uses the Unicode model to represent the fundamental text. XML, defines a set of rules for encrypting any data of information (documents) in the format of both human-readable and machine-readable. XML Document encloses three important parts such as XML Declaration, DTD and XML Instance. Example of XML instance is given below ${ }^{3}$. 


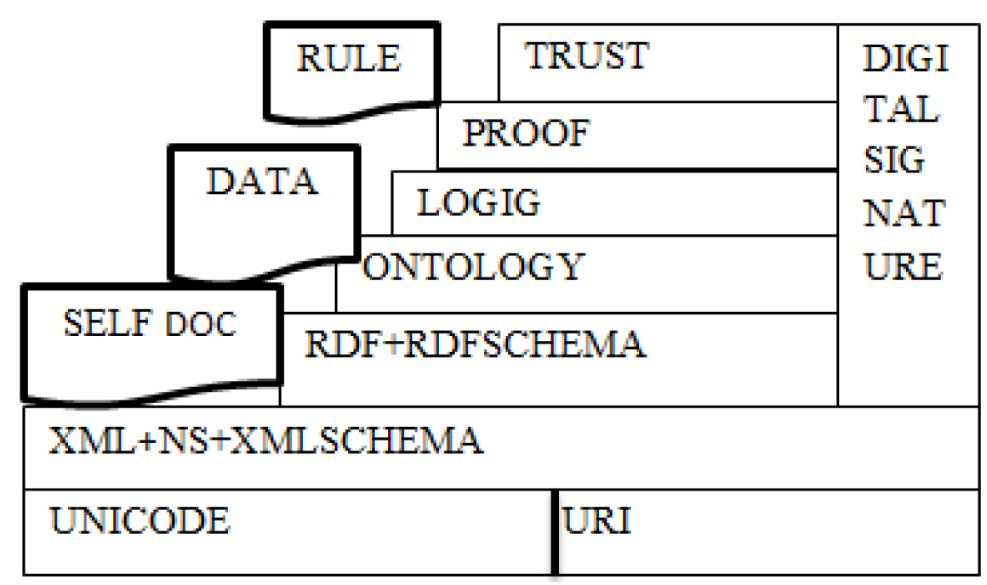

Figure 1.1 Architecture of semantic web.

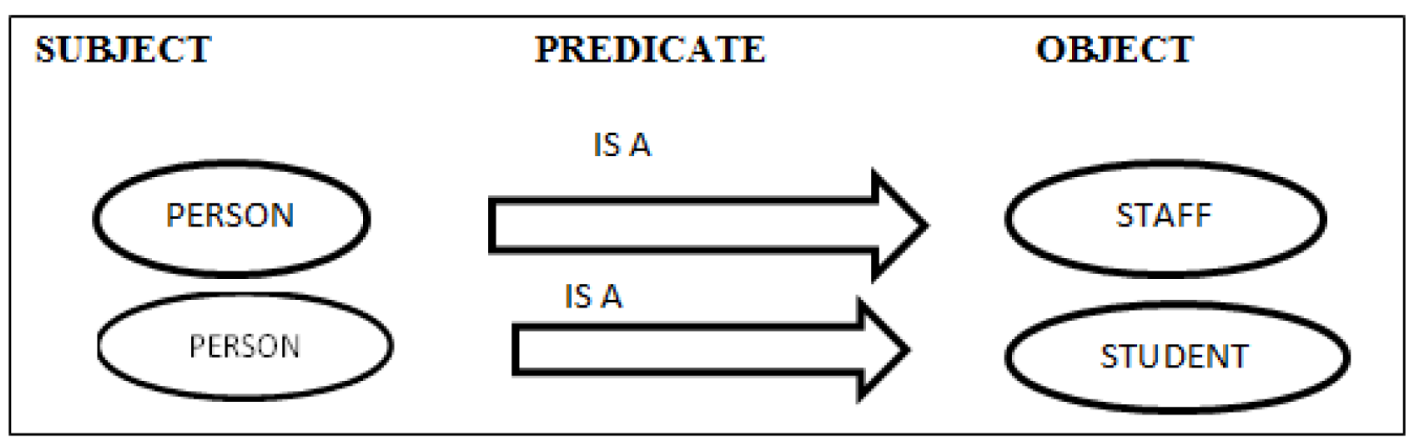

Figure 1.2 Representation of sementic Data.

$$
\begin{aligned}
& <\text { ?XMLversion="1.0"? > } \\
& <\text { library }> \\
& <\text { author }>\text { Balagurusamy </author }> \\
& <\text { title }>\text { Structure of C Program }<\text { /title }> \\
& <\text { publisher }>\text { TATA }<\text { /publisher }> \\
& <\text { library }>
\end{aligned}
$$

RDF is originally designed based on the Meta data model. It signifies the details of subject, property, value. RDF has some restricted vocabulary. RDFS schema contains much more vocabulary compare to RDF. RDF, RDFS everything is based on Triple. The example of RDF schema is given below

$$
\begin{aligned}
& \text { <rdfs:Classrdf:ID="Person"> } \\
& \text { <rdfs:SubClassOf="Staff"/> } \\
& \text { <rdfs:SubClassOf="student"/> } \\
& \text { </rdfs:Class }>
\end{aligned}
$$

Figure 1.2 represents the way to builddata in semantic web. Ontology is exemplified by the high level language OIL (Ontology Interference Layer).OIL is converted into
RDF/XML using Parser. OWL is greatest way to denote the ontologies. There is a lot of Ontology based tools has been introduced ${ }^{4}$.

Protégé is one of main ontology based tool. It is open source editor. Jess is a rule engine and scripting environment. Jess used to build Java software based on declarative rules. Jess is little, light, and one of the highest rule engine. Its most powerful scripting language to contact all Java's APIs (Application programming interfaces).Third Jena is a java based semantic web application tools. It contain programmatic environment like RDF, RDFS, OWL and also including rule based engine. This is also an open source editor. Fourth joseki is a sever based engine for RDF models. Each model has URL. They can access by HTTP GET Method and it is also part of JENA Framework. Simple representation of ontology based OWL is given below.

<owl:Classrdf:ID="Course" >

$<$ rdfs:subclassOfrdfs:resource="Material" $>$

.......

$</$ owl:Class $>$ 


\section{Implementing Semantic based E-learning}

Our proposed semantic web based e learning system model is based on the concept hierarchy.

Figure 2.1 shows the details of person classis categorized in to two effects: Teacher and Student. The students have two types of content such as learning content and assessment. Learning services provide the details of registration, course material, etc. Assessment is conducted as test, quiz, and assignment agreed to the staff'.

The Tutor has a control to authenticate the student or learners. They have full honors to add or remove any learner from the particular group. Instructor Monitor the student based on the accompanying test, workouts, projects, Quiz etc., all these type of evidence is stored in the database. Then next instructor also formulates the list of course material, content of the each course material for the lecture. They can use any type teaching tools ${ }^{5}$.

Further student can record her/his details. The communication between the tutor and learner may be synchronous or asynchronous. Video conference is a greatest two way communication. Learner side any registered student can login to the group or a novel user can sign up for the equal. Learner and tutor use any type of teaching method. Learner also posts their question in the particular page. Those queries are automatically forward to the tutor.
This Proposed work implements the class concept based algorithm method for semantic web technologies using PROTÉGÉ tool. First creates an ontology based Domain for e-learning concept. The two main class domains in this proposed model are Tutor and Student. Second add sub class (child class) data and its property of these two main classes tutor and student. The tutor Parent class contains the sub class (child) details about the personal details of tutor, Monitor student presentation, Course Material etc. Add required number of subclass in the parent classes. Like that the subclass of monitor student performance class also includes more child class details like quiz, assessment, project details etc. The equal way adds the more subclass and its details.

Next generates one more main domain class for student. It contains the details of personal information and basic eligibility of student. The learner (student) class added a few more subclass like registration, tutorial, semantic search, course work etc. Next add the different property for the each Tutor and Student domain. Then creates the number of individuals, object property in Protégé tool.

Figure 2.2 represents all the domains of e-learning concept using class hierarchy method. After implementing all the details in Protégé tools, user can set a rule based concept using SPARQL query for collecting result like number of student registration, number of test

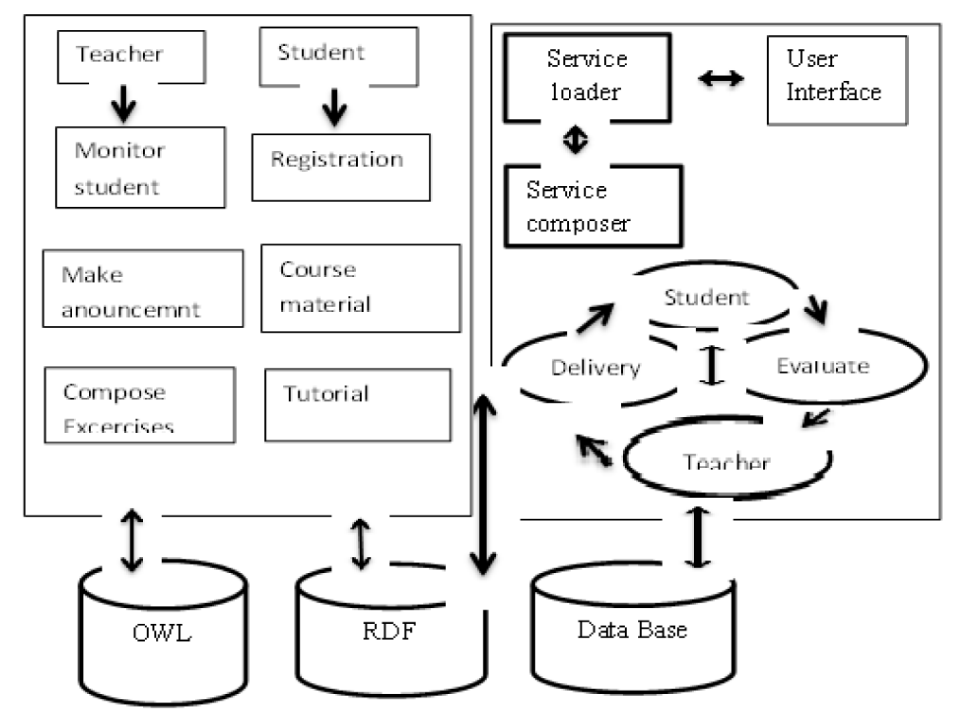

Figure 2.1 Semantic web architecture. 


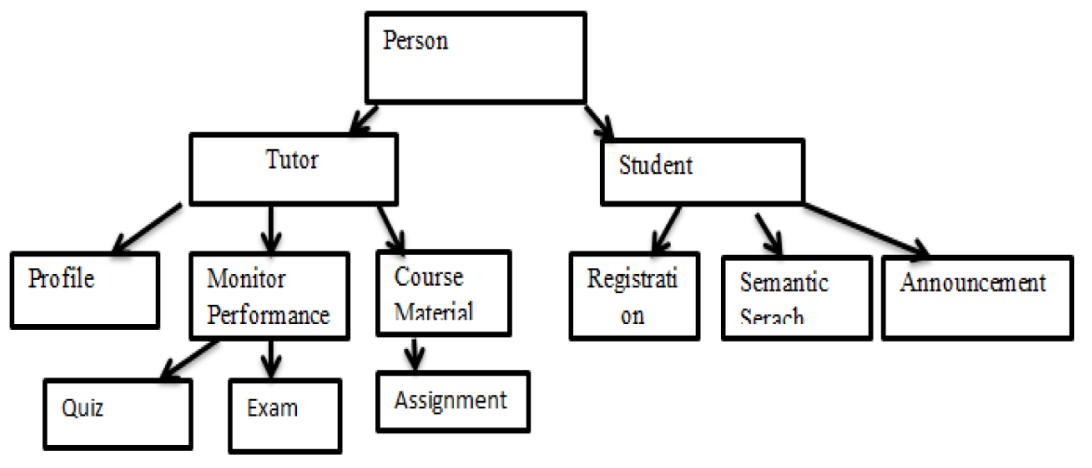

Figure 2.2 Semantic web architecture.

conducted by the particular tutor etc ${ }^{6}$. Like that implement any SPARQL query to extracting the certain information.

<owl:Classrdf:ID="http://www.w3.org/1999/02/22rdf-Student-ns\#">

$<$ rdfs:subClassOfrdfs:resource="StRegistration" $>$

$<$ owl:Restriction $>$

$<$ owl:on Propertyrdf: resource $=$ "http://www. w3.org/1999/02/22-rdf-Student-ns “/>

$<$ owl:someValuesFromrdf:resource="\&xsd;Integer"/>

$</$ owl:Restriction $>$

$</$ rdfs:subClassOf $>$

$<$ /owl:class $>$

\section{Conclusion}

This Paper proposed class concept hierarchy methods for ontology based e-learning application using semantic techniques in ontology based PROTÉGÉ tools. And it returns various information using SPARQL query and reduce the drawback of keyword and document based techniques search model. This proposed work also integrated into Oracle, SQL, and Java Language. In Future implement these concepts of semantic web based e-learning through the mobile device using JENA. It is best suitable tool for creation of any mobile based application. This proposed work can also be integrated into Oracle, SQL, and Java Language.

\section{References}

1. Ghaleb F, Daoud S, Hasna A, ALJa'am JM, El-Seoud SA, El-Sofany H. E-learning model based on semantic web technology. International Journal of Computing \& Information Sciences. 2006 Aug; 4(2):

2. Ahmed S, Parsons D. ThinknLearn: An Ontology-Driven Mobile Web Application For Science Enquiry Based Learning. 7th International Conference on Information Technology and Applications (ICITA 2011);

3. Sheeba T, Begum SH, Bernard MJ. Semantic Web to E-Learning Content. International Journal of Advanced Research in Computer Science and Software Engineering. 2012 Oct; 2(10):

4. Khozooyi N, Nafiseseyedi, Malekhoseini R. Ontologybased e-learning. IJCSITS. 2012 Aug; 2(4):

5. Dwivedi A, Bawankan A. Development of Semantic E-Learning Web using Protégé. Conference on Advances in Communication and Control Systems; 2013.

6. Tulasi L, Rao MS, Gouda GR. Study of E-learning Information Retrieval Model based on Ontology. Int J Comput Appl. 2013 Jan; 61(17): 